\title{
Specific Combining Ability analysis for Grain Yield and its Components in Post Rainy Sorghum
}

\author{
Shailesh M. Gawande*, Vikram V. Kalpande and Sunil B. Thawari
}

All India Coordinated Sorghum Improvement Project, Akola Centre, Sorghum Research Unit, Dr. Panjabrao Deshmukh Krish iVidyapeeth, Akola - 444001 (M.S.), India

*Corresponding author

\section{A B S T R A C T}

K e y w o r d s
Combining ability
analysis, Line x
tester, SCA,
Sorghum, Standard
heterosis

Five lines and ten testers were crossed in line $\mathrm{x}$ tester design to produce 50 cross combinations and were evaluated along with the check for grain yield and its components for combining ability analysis. The study revealed that out of the fifty hybrids, thirteen hybrids revealed positive significant SCA effects along with positive significant standard heterosis for grain yield per plant. Hybrid AKRMS-80-1A (39) x PKV-Kranti recorded the highest positive significant SCA effects $(25.93 * *)$ along with positive significant standard heterosis $(19.72 * *)$ for grain yield/plant. All these crosses need to be evaluated on large scale multilocation and multiseason trials to find out the most stable cross combination for higher grain yield in rabi sorghum.

\section{Introduction}

Post rainy (Rabi) sorghum is dual purpose crop with best grain quality and fodder quality and hence fetches good market price. Post rainy sorghum is mostly taken on the residual soil moisture. The study was undertaken to estimate the specific combining ability effects in post rainy sorghum hybrids which in turn helped in identification of the potential cross combinations for yield and its components in post rainy sorghum. Promising cross combinations were sorted out based on the specific combining ability along with the desirable and significant standard heterosis for grain yield per plant.

\section{Materials and Methods}

The experimental material comprised of five male sterile lines viz., AKRMS-66-2A(38), AKRMS-66-2A(40), AKRMS-66-2-3A, AKRMS-80-1A(39) and AKRMS-80-11A(62) and ten testers viz., SLR-136, SLR137, Elangovan-35, AKSV-252, Rb-Local-11-sel-1, Rb-Local-5 (Bold), RSV-962,AKSV330, PKV-Kranti and AKSV-370. These fifteen genotypes were crossed in line $\mathrm{x}$ tester 
fashion. Fifteen parents and their resulting 50 hybrids along with one standard check $\mathrm{CSH}$ 19R were sown at Sorghum Research Unit, Dr. P.D.K.V. Akola in randomized block design with three replications. The observations were recorded on five randomly selected plants per plot per replication for plant height $(\mathrm{cm})$, panicle length $(\mathrm{cm})$, panicle breadth $(\mathrm{cm})$, number of primaries/ panicle, number of grains/ panicle, 1000 seed weight (g) and grain yield/ plant $(\mathrm{g})$. The standard heterosis was estimated as per cent increase or decrease of the mean of $F_{1}$ over the value of the standard check CSH 19 R. The data on all the above characters were subjected to combining ability analysis by following Kempthorne (1957) method.

\section{Results and Discussion}

Analysis of variance showed that the mean squares due to genotypes were highly significant for all the traits studied under this investigation (Table 1). This indicated the presence of substantial genetic variability for these characters. Further partitioning of genotypic variance into components viz., parents, hybrids and parents vs. hybrids revealed that the parents differed among themselves significantly for grain yield per plant. Analysis of variance for combining ability is presented in Table 2. It is revealed that crosses exhibited significant variation for grain yield as well as all the component traits. The male $\mathrm{x}$ female interaction was also significant. Among the 50 hybrids in the present investigation, thirteen cross combinations exhibited positive SCA effects for grain yield per plant along with some of the component characters (Table 3). The hybrid AKRMS-80-1A x PKV-Kranti $(25.93 * *) \quad$ exhibited highest positive significant SCA effects for grain yield per plant along with all other component characters.
The second hybrid AKRMS-66-2-3A x SLR136 exhibited desirable significant SCA effects for grain yield per plant (20.37**) along with all other component characters except plant height. The SCA effects were $8.55^{* *}$ for number of primaries, $2.73^{* *}$ for panicle length, $0.71 * *$ for panicle breadth and $4.22 * *$ for 1000 seed weight.

Third cross AKRMS-80-1A (39) $\mathrm{x}$ Elangovan-35 exhibited positive significant SCA effects for grain yield per plant $(13.43 * *)$ along with all other component characters except for panicle length and panicle breadth. Total thirteen crosses exhibited positive significant SCA effects along with positive significant standard heterosis for grain yield per plant.

Ghorade et al., (2016) reported ten promising cross combination based on positive significant SCA effects for grain yield per plant.

Kalpande et al., (2016) also reported three promising crosses based on positive significant SCA effects for grain yield per plant. Thus it was also observed that thirteen crosses exhibited positive significant SCA effects accompanied with positive significant standard heterosis for grain yield per plant. It is very well known that if SCA variance, which is a measure of non-additive genetic variance, is high for a character and also observed heterosis is also high, such crosses can be utilized for commercial exploitation of heterosis.

Similarly, it was also observed from the Table 3 that high heterotic crosses exhibited significant SCA effects with higher per se performance. Similar relationship was also noticed by Prabhakar (2013) in rabi sorghum. However Patil and Bapat (1991) reported that high SCA was not reflected in high heterosis. 
Prakash et al., (2010), Prabhakar et al., (2013), Ghorade et al., (2014 $\left.{ }^{\mathrm{b}}\right)$ and Kalpande et al., $\left(2015^{\mathrm{c}}\right)$ also reported such promising crosses based on SCA, heterosis and mean performance.

Gunjal (2014) also reported fourteen promising crosses with positive significant SCA effects along with positive significant standard heterosis in his study.
Kalpande et al., $\left(2016^{\mathrm{d}}\right)$ reported seventeen promising cross combinations based on positive significant SCA effects for grain yield per plant along with some of the component characters.

Ghorade et al., (2018) reported the cross combination AKMS 90 A x AKR 337 with highest significant standard heterosis as well as highest significant SCA effects for grain yield per plant.

Table.1 Analysis of variance of parents and hybrids for various characters under Line $\mathrm{x}$ Tester analysis

\begin{tabular}{|c|c|c|c|c|c|c|c|c|}
\hline $\begin{array}{l}\text { Source of } \\
\text { Variation }\end{array}$ & d.f. & $\begin{array}{c}\text { Plant } \\
\text { Height }(\mathrm{cm})\end{array}$ & $\begin{array}{c}\text { Number of } \\
\text { primaries } \\
\text { per cob }\end{array}$ & $\begin{array}{c}\text { Panicle } \\
\text { Length } \\
(\mathrm{cm})\end{array}$ & $\begin{array}{l}\text { Panicle } \\
\text { breadth } \\
\text { (cm) }\end{array}$ & $\begin{array}{c}\text { Number of } \\
\text { Grains/ } \\
\text { Panicle }\end{array}$ & $\begin{array}{l}1000 \text { Seed } \\
\text { Weight }(g)\end{array}$ & $\begin{array}{c}\text { Grain } \\
\text { Yield/ } \\
\text { Plant (g) }\end{array}$ \\
\hline Replications & 2 & 9.89 & 2.72 & 1.68 & 0.11 & 814.74 & 2.27 & 1.26 \\
\hline Genotypes & 64 & $3340.27 * *$ & $263.78 * *$ & $11.28 * *$ & $1.22 * *$ & $571863.91 * *$ & $85.30 * *$ & $566.34 * *$ \\
\hline Parents & 14 & $2741.95 * *$ & $174.46^{* *}$ & $8.96 * *$ & $0.28^{*}$ & $215933.56 * *$ & $83.48 * *$ & $296.44 * *$ \\
\hline Females & 4 & $154.44 * *$ & $401.16^{* *}$ & $9.99 * *$ & 0.31 & $233073.87 * *$ & $121.34 * *$ & $598.69 * *$ \\
\hline Males & 9 & $547.24 * *$ & $84.59 * *$ & $9.26 * *$ & $0.29 *$ & $226876.72 * *$ & $74.31 * *$ & $165.29 * *$ \\
\hline $\begin{array}{l}\text { Females vs } \\
\text { Males }\end{array}$ & 1 & $32844.3617 * *$ & $76.54 * *$ & 2.19 & 0.007 & $48883.86 * *$ & $14.48 *$ & $267.73 * *$ \\
\hline Hybrids & 49 & $3565.7858 * *$ & $277.41 * *$ & $11.58 * *$ & $1.51 * *$ & $678127.736 * *$ & $83.41 * *$ & $651.57 * *$ \\
\hline $\begin{array}{l}\text { Parents vs } \\
\text { Hybrids }\end{array}$ & 1 & $666.56^{* *}$ & $846.26 * *$ & $29.02 * *$ & 0.015 & $347961.26 * *$ & $203.78 * *$ & $168.86 * *$ \\
\hline Error & 128 & 20.23 & 6.57 & 1.98 & 0.14 & 4526.13 & 2.49 & 7.62 \\
\hline
\end{tabular}

Table.2 Analysis of variance for combining ability for various characters

\begin{tabular}{|c|c|c|c|c|c|c|c|c|}
\hline $\begin{array}{l}\text { Source of } \\
\text { Variation }\end{array}$ & d.f. & $\begin{array}{c}\text { Plant } \\
\text { Height } \\
\text { (cm) }\end{array}$ & $\begin{array}{l}\text { Number } \\
\text { of } \\
\text { Primaries } \\
\text { per cob }\end{array}$ & $\begin{array}{c}\text { Panicle } \\
\text { Length } \\
\text { (cm) }\end{array}$ & $\begin{array}{c}\text { Panicle } \\
\text { Breadth } \\
\quad(\mathrm{cm})\end{array}$ & $\begin{array}{c}\text { Number } \\
\text { of } \\
\text { Grains/ } \\
\text { Panicle }\end{array}$ & $\begin{array}{c}1000 \\
\text { Seed } \\
\text { Weight } \\
\text { (g) }\end{array}$ & $\begin{array}{c}\text { Grain } \\
\text { Yield/ } \\
\text { Plant (g) }\end{array}$ \\
\hline Replications & 2 & 11.198 & 15.020 & 1.958 & 0.138 & 6158.657 & 2.382 & 2.064 \\
\hline Crosses & 49 & $3565.786 * *$ & $277.418 * *$ & $11.585 * *$ & $1.516 * *$ & $678127.800 * *$ & $83.414 * *$ & $651.575 * *$ \\
\hline Lines & 4 & $12546.210 * *$ & 416.333 & 13.590 & 1.353 & $2222591.000 * *$ & 51.456 & $2291.907 * *$ \\
\hline Testers & 9 & 3579.973 & 355.456 & 9.845 & 1.701 & 857341.700 & 149.956 & 881.244 \\
\hline Line $x$ Teste & 36 & $2564.414 * *$ & $242.474 * *$ & $11.797 * *$ & $1.488 * *$ & $461717.200 * *$ & $70.330 * *$ & $411.899 * *$ \\
\hline Error & 98 & 20.770 & 7.347 & 2.122 & 0.164 & 3670.258 & 2.538 & 8.735 \\
\hline
\end{tabular}

* - significant at $5 \%$ level of significance ** - significant at $1 \%$ level of significance 
Table.3 SCA effects of promising hybrids for grain yield per plant

\begin{tabular}{|c|c|c|c|c|c|c|}
\hline S.N & Crosses & $\begin{array}{c}\text { Mean } \\
\text { for } \\
\text { Grain } \\
\text { yield } \\
\text { / plant } \\
\text { (g) }\end{array}$ & $\begin{array}{l}\text { SCA } \\
\text { effects for } \\
\text { grain yield }\end{array}$ & $\begin{array}{l}\text { Standard } \\
\text { Heterosis } \\
\text { for grain } \\
\text { yield }(\%)\end{array}$ & $\begin{array}{l}\text { GCA effect with } \\
\text { type of parents for } \\
\text { grain yield }\end{array}$ & $\begin{array}{c}\text { Desirable significant SCA effects for } \\
\text { component traits }\end{array}$ \\
\hline 1 & $\begin{array}{l}\text { AKRMS-80-1A(39) } \\
\text { X PKV-Kranti }\end{array}$ & 66.42 & $25.93 * *$ & $19.72 * *$ & $\begin{array}{l}-2.72 * * \\
\text { X }-4.68 * * \\
\text { L L }\end{array}$ & $\begin{array}{l}\text { plant height, panicle length,, panicle } \\
\text { breadth, number of primaries/ panicle, } \\
\text { number of grains/ panicle, } 1000 \text { seed } \\
\text { weight }\end{array}$ \\
\hline 2 & $\begin{array}{l}\text { AKRMS-66-2-3A X } \\
\text { SLR-136 }\end{array}$ & 69.17 & $20.37 * *$ & $24.67 * *$ & $\begin{array}{l}3.56 * * \mathrm{X}-2.65 * * \\
\mathrm{HL}\end{array}$ & $\begin{array}{l}\text { panicle length,, panicle breadth, number of } \\
\text { primaries/ panicle, number of grains/ } \\
\text { panicle, } 1000 \text { seed weight }\end{array}$ \\
\hline 3 & $\begin{array}{l}\text { AKRMS-80-1A(39) } \\
\text { X Elangovan-35 }\end{array}$ & 66.76 & $13.43 * *$ & $20.32 * *$ & $\begin{array}{l}-2.72 * * \times 8.15 * * \\
\mathrm{~L} \mathrm{H}\end{array}$ & $\begin{array}{l}\text { plant height, number of primaries/ panicle, } \\
\text { number of grains/ panicle, } 1000 \text { seed } \\
\text { weight }\end{array}$ \\
\hline 4 & $\begin{array}{l}\text { AKRMS-80-1- } \\
\text { 1A(62) x } \\
\text { Rb-Local-1-1-sel-1 }\end{array}$ & 67.46 & $13.05 * *$ & $21.59 * *$ & $\begin{array}{l}-9.14 * * \\
\text { X } 15.66^{* *} \\
\text { L H }\end{array}$ & $\begin{array}{l}\text { plant height, panicle length,, panicle } \\
\text { breadth, number of primaries/ panicle, } \\
\text { number of grains/ panicle, } 1000 \text { seed } \\
\text { weight }\end{array}$ \\
\hline 5 & $\begin{array}{l}\text { AKRMS-66-2-3A X } \\
\text { Rb- Local -5(Bold)) }\end{array}$ & 63.42 & $10.44 * *$ & $14.30 * *$ & $\begin{array}{l}3.56^{* *} \times 1.52 * \\
\mathrm{H} \mathrm{H}\end{array}$ & $\begin{array}{l}\text { panicle length, number of primaries/ } \\
\text { panicle, number of grains/ panicle }\end{array}$ \\
\hline 6 & $\begin{array}{l}\text { AKRMS-66-2-3A X } \\
\text { AKSV-370 }\end{array}$ & 65.36 & $10.38 * *$ & $17.80 * *$ & $\begin{array}{l}3.56^{* *} \times 3.52 * * \\
\mathrm{H} \mathrm{H}\end{array}$ & number of primaries/ panicle \\
\hline 7 & $\begin{array}{l}\text { AKRMS-66-2A(38) } \\
\text { X } \\
\text { SLR-137 }\end{array}$ & 72.21 & $10.22 * *$ & $30.15^{* *}$ & $\begin{array}{l}13.30 * * \times 0.17 \\
\mathrm{H} \mathrm{H}\end{array}$ & $\begin{array}{l}\text { plant height, panicle length,, panicle } \\
\text { breadth, number of primaries/ panicle, } \\
\text { number of grains/ panicle, } 1000 \text { seed } \\
\text { weight }\end{array}$ \\
\hline 8 & $\begin{array}{l}\text { AKRMS-66-2A(40) } \\
\mathrm{X} \\
\text { Rb-Local-1-1-sel-1 }\end{array}$ & 68.00 & $9.45 * *$ & $22.57 * *$ & $\begin{array}{l}-5.01 * * \quad \quad \mathrm{X} \\
15.66^{* *} \\
\text { L H }\end{array}$ & panicle breadth, 1000 seed weight \\
\hline 9 & $\begin{array}{l}\text { AKRMS-66-2A(38) } \\
\text { X } \\
\text { RSV-962 }\end{array}$ & 65.26 & $9.32 * *$ & $17.61 * *$ & $\begin{array}{l}13.30 * * \quad \mathrm{X}- \\
5.26 * * \\
\mathrm{H} \mathrm{L}\end{array}$ & $\begin{array}{l}\text { plant height, number of primaries/ panicle, } \\
\text { number of grains/ panicle, } 1000 \text { seed } \\
\text { weight }\end{array}$ \\
\hline 10 & $\begin{array}{l}\text { AKRMS-80-1A(39) } \\
\text { X } \\
\text { Rb-Local-1-1-sel-1 }\end{array}$ & 69.87 & $9.04 * *$ & $25.93 * *$ & $\begin{array}{ll}-2.52 * * & X \\
15.66^{* *} & \\
\text { L H } & \end{array}$ & $\begin{array}{l}\text { plant height, panicle length,, panicle } \\
\text { breadth, number of primaries/ panicle, } \\
\text { number of grains/ panicle, } 1000 \text { seed } \\
\text { weight }\end{array}$ \\
\hline 11 & $\begin{array}{l}\text { AKRMS-66-2-3A X } \\
\text { Elangovan-35 }\end{array}$ & 68.52 & $8.91 * *$ & $23.50 * *$ & $\begin{array}{l}3.56^{* *} \times 8.15^{* * *} \\
\mathrm{H} \mathrm{H}\end{array}$ & $\begin{array}{l}\text { plant height, panicle breadth, number of } \\
\text { primaries/ panicle, number of grains/ } \\
\text { panicle, } 1000 \text { seed weight }\end{array}$ \\
\hline 12 & $\begin{array}{l}\text { AKRMS-66-2A(38) } \\
\text { X } \\
\text { Rb-Local-5(Bold) }\end{array}$ & 68.92 & $6.19 * *$ & $24.21 * *$ & $\begin{array}{l}13.30^{* *} \text { X } 1.52^{*} \\
\mathrm{H} \mathrm{H}\end{array}$ & $\begin{array}{l}\text { plant height, panicle length,, number of } \\
\text { primaries/ panicle, number of grains/ } \\
\text { panicle }\end{array}$ \\
\hline 13 & $\begin{array}{l}\text { AKRMS-66-2A(38) } \\
x \\
\text { AKSV-370 }\end{array}$ & 69.91 & $5.19 * *$ & $26.01 * *$ & $\begin{array}{l}13.30 * * \\
3.52 * * \\
\mathrm{H} \mathrm{H}\end{array}$ & $\begin{array}{l}\text { panicle breadth, number of primaries/ } \\
\text { panicle, number of grains/ panicle, } 1000 \\
\text { seed weight }\end{array}$ \\
\hline
\end{tabular}

* - significant at $5 \%$ level of significance ** - significant at $1 \%$ level of significance 
For grain yield per plant, out of the total thirteen hybrids, six hybrids involved high $\mathrm{x}$ low type of GCA value of parents involved in the crosses while six cross combination involved high $\mathrm{x}$ high type of GCA value of the parents and one cross combination involved low x low type of GCA value of the parents

Ravindrababu et al., (2001) and Ghorade et al., (2014) also reported that some of the promising hybrids involved high $\mathrm{x}$ low GCA parental lines. However Hariprasanna et al., (2012) reported that some of the crosses with positive significant SCA for grain yield involved even low $\mathrm{x}$ low combination of parents.

Gunjal (2014) also reported that out of the total sixteen promising hybrids, eleven hybrids involved high $\mathrm{x}$ low type of GCA value of parents involved in the crosses while five cross combination involved low $\mathrm{x}$ low type of GCA value of the parents.

Thus it was concluded from the present study that total thirteen cross combinations recorded positive significant SCA effects along with positive significant standard heterosis for grain yield per plant and hence appeared to be best for further exploitation. These crosses need to be evaluated on large scale multilocation and multiseason trials to find out the most stable cross combination for higher grain yield in rabi sorghum.

\section{References}

Ghorade et al., (2014). Combining ability analysis for drought tolerance and grain yield in rabi sorghum. International Journal of Agricultural Sciences 10(1):344-347.

Ghorade et al., 2016). Specific combining ability for grain yield and yield components in rabi sorghum under drought condition. National Conference on Genetics and Cytogenetisc, 1-3 February, Dharwad, India, pp-67-68.

Ghorade et al., (2018). Assessment of newly developed diverse parental lines in sorghum through combining ability. Int. J. Curr. Microbiol. App. Sci. 6: 19921998.

Gunjal, S.M. (2014). Heterosis and combining ability studies in rabi sorghum. M.Sc. (Agri.). Unpub. Thesis, Panjabrao Deshmukh Krishi Vidyapeeth, Akola.

Hariprasanna, K., Rajendrakumar P. and Pati IJ.V. (2012).Parental selection for high heterosis in sorghum (Sorghum bicolor (L.) Moench)-Combining ability, heterosis and their relationship. Crop Res., 44(3):400-408.

Kalpande et al., (2015).Combining ability analysis for grain and fodder yield in post rainy sorghum. Plant Archives, Vol.15 No.1, 2015 pp.105-107.

Kalpandeet.al. (2016). Specific combining ability effects for grain yield and yield contributing traits in post rainy sorghum. Int. Conference on Plant Res. and Resource Mgt., 11-13 February 2016, Baramati (MS) India, pp-63.

Kempthorn O. (1957). An Introduction to genetic statistics. John Willey and Sons. Increased. New York, 468-470

Patil, F.B. and Bapat D.R. (1991). Stability parameters in sorghum. J. Maharashtra Agric. Univ., 16(1): 13-15.

Prabhakar et al., (2013).Combining ability of new parental lines for flowering, maturity and grain yield in rabi sorghum. Electronic J. of Plant Breeding, 4(3): 1241-1218.

Prakash et al., 2010). Combining ability for fodder yield and its components in sorghum (Sorghum bicolor (L.) Moench). Electronic Journal of Plant Breeding.1 (2):140-144. 


\section{How to cite this article:}

Shailesh M. Gawande, Vikram V. Kalpande and Sunil B. Thawari. 2020. Specific Combining Ability analysis for Grain Yield and its Components in Post Rainy Sorghum. Int.J.Curr.Microbiol.App.Sci. 9(08): 2304-2309.

doi: https://doi.org/10.20546/ijcmas.2020.908.264 